\title{
Towards a Rigorous Treatment of the Jeans-Landau-Teller Method for the Energy Exchanges of Harmonic Oscillators
}

\author{
Andrea Carati ${ }^{1}$, Giancarlo Benettin ${ }^{1,2}$ and Luigi Galgani ${ }^{3}$ \\ ${ }^{1}$ Università di Padova, Dipartimento di Matematica Pura e Applicata, Via Belzoni 7, J-35121 \\ Padova, Italy \\ ${ }^{2}$ INFM, Università di Padova, Via Belzoni 7, J-35121 Padova, Italy \\ 3 Università di Milano, Dipartimento di Matematica, Via Saldini 50, J-20133 Milano, Italy
}

Received July 1, 1991; in revised form March 24, 1992

\begin{abstract}
For classical Hamiltonian systems containing an harmonic oscillator of high frequency, one has the problem of controlling the energy exchange between the oscillator and the remaining "slow" degrees of freedom; under very general conditions, such an exchange turns out to be exponentially small with the frequency of the oscillator. In the Jeans-Landau-Teller method, one aims to prove the exponential dependence, and to estimate the coefficient of the exponential, by exploiting the analyticity properties of the solution of the differential equations describing the motion of the system. However, in practice, since the exact solution is not known, such properties are inferred from those of an approximate solution, with no control of the difference; this fact might a priori even invalidate the exponential dependence itself. In the present paper a rigorous treatment is given, for a particular model of interest in the domain of atomic collisions, by keeping control of the difference between the exact and the approximate solution.
\end{abstract}

\section{Introduction}

The problem of estimating the exchanges of energy for systems containing harmonic oscillators of high frequency is a very relevant one in many domains of physics. A rigorous mathematical treatment was given, rather recently, by Neishtadt [1] and by Benettin, Galgani and Giorgilli [2, 3], by adapting the methods of classical perturbation theory developed by Nekhoroshev [4, 5]. A typical result is that the exchange of energy between a system of harmonic oscillators of the same angular frequency $\omega$ and another system is, under quite general conditions, exponentially small with $\omega$ :

$$
\Delta E \leqq C e^{-a \omega}, \quad C, a \geqq 0,
$$

if $\omega$ is sufficiently large. Unfortunately, as is typical of perturbation theory, the estimates one gets for the constants, in particular for $a$, which is the most relevant one, are terribly pessimistic and, as could be seen by numerical computations [6] (see also [7] and the papers there quoted), very far from being realistic. Moreover, 
the exponential law comes out in a somehow unnatural way, as the result of a rough estimate for the remainder of an asymptotic series; in other words, the mathematical mechanism leading to the exponential law is not really transparent there.

On the other hand, qualitative results of the form (1.1) are well known to researchers in several domains of applied physics, such as atomic collisions $[8,7]$ and plasmas [9]. The method used, which goes back to Jeans [10, 11] and to Landau and Teller [12] (and perhaps even to Rayleigh), is based on the fact that the energy exchange $\Delta E$ is simply related to the Fourier transform of a convenient analytic function $u(t)$, directly constructed from the solution of the differential equations describing the system (see below). Thus, the exponential law comes out rather directly as a consequence of the standard estimate on Fourier transforms; in particular, the distance of the closest singularity of $u$ from the real axis plays a crucial role, as it gives the coefficient $a$. However, we were not able to find in the literature a rigorous implementation of this scheme. The problem is that usually one is not able to control the singularities of $u$ itself, since it depends on the exact solution, which is not known; so one replaces the exact solution by a convenient first order approximation, supposing that the corresponding functions have essentially the same singularities; but a clear justification of this point is, as far as we know, lacking. Moreover, one is confronted with the general problem of understanding the relationship between the two methods, namely that of classical perturbation theory and that of Jeans and Landau-Teller.

In the present paper we address the first of the two problems mentioned above, at least in the case of a particular model. In fact, we prove rigorously the exponential estimate (1.1) by the Jeans-Landau-Teller method, by controlling the relevant singularities for the exact function $u$; this is done in a particular case for a model of interest in the problem of atomic collisions. We hope to be able in the future to give a general theorem of such a type, and also to give the general connection with the method of classical perturbation theory.

In Sect. 2 we briefly recall the model and the standard heuristic method. In Sect. 3 we formulate our main proposition, and deduce from it a corollary with the exponential estimate. The proofs are given in Sects. 4 and 5. In the Appendix, a short description of the Hamiltonian considered here, introduced by Rapp [8], is given.

\section{The Jeans-Landau-Teller Method for a Particular Model}

The model we study is a Hamiltonian system with two degrees of freedom, with Hamiltonian

$$
H\left(x, y, p_{x}, p_{y}\right)=\frac{1}{2}\left(p_{y}^{2}+\omega^{2} y^{2}\right)+\frac{1}{2} p_{x}^{2}+e^{-x}(1+y),
$$

and variables $\left(x, y, p_{x}, p_{y}\right) \in \mathbb{R}^{4}$. Such a Hamiltonian describes, in some approximation [8], the collinear collision of a diatomic vibrating molecule, with proper angular frequency $\omega$, with a point-mass coming from $+\infty$, the point-mass and the closer atom of the molecule being supposed to interact via a repulsive exponential potential; equivalently, one can think of a molecule colliding with a fixed wall (for more details, see the Appendix). The corresponding equations of motion are

Denote by

$$
\ddot{x}-e^{-x}=y e^{-x}, \quad \ddot{y}+\omega^{2} y=-e^{-x} .
$$




$$
E^{x}=\frac{1}{2} \dot{x}^{2}+e^{-x}, \quad E^{y}=\frac{1}{2}\left(\dot{y}^{2}+\omega^{2} y^{2}\right)
$$

the unperturbed energies of the particle and of the oscillator respectively. We are interested in the energy acquired by the oscillator after the collision, more precisely in the quantity

$$
\Delta E=\lim _{\substack{t_{0} \rightarrow-\infty \\ t \rightarrow \infty}}\left(E^{y}(t)-E^{y}\left(t_{0}\right)\right)
$$

The method used is essentially as follows: denote by $x_{\omega}(t), y_{\omega}(t)$ the solution of $(2.2)$, and introduce the complex amplitude $A_{\omega}=\dot{y}_{\omega}+i \omega y_{\omega}$, so one has that $E^{y}=\frac{1}{2}\left|A_{\omega}\right|^{2}$. From the second of (2.2) one easily obtains the integral expression

$$
A_{\omega}(t)=A_{\omega}\left(t_{0}\right) e^{i \omega\left(t-t_{0}\right)}-\int_{t_{0}}^{t} e^{-x_{\omega}\left(t^{\prime}\right)} e^{i \omega\left(t-t^{\prime}\right)} d t^{\prime},
$$

so that one gets

$$
E^{y}(t)-E^{y}\left(t_{0}\right)=\frac{1}{2}\left|\int_{t_{0}}^{t} e^{-x_{\omega}(t)} e^{i \omega t} d t\right|^{2}+\left|A\left(t_{0}\right)\right|\left|\int_{t_{0}}^{t} e^{-x_{\omega}(t)} e^{i \omega t} d t\right| \cos \varphi_{0}
$$

$\varphi_{0}$ being a suitable phase. Introducing now the Fourier transform of $e^{-x_{\omega}(t)}$ :

$$
\hat{u}_{\omega}(v)=\int_{\mathbb{R}} e^{-x_{\omega}(t)} e^{i v t} d t
$$

( $v$ is here conjugate to $t$, while $\omega$ is a parameter), from (2.4) and (2.5) one gets for $\Delta E$ an expression of the form

$$
\Delta E=\frac{1}{2}\left|\hat{u}_{\omega}(\omega)\right|^{2}+|A(-\infty)|\left|\hat{u}_{\omega}(\omega)\right| \cos \varphi_{\infty},
$$

with suitable $\varphi_{\infty}$.

The problem is thus to estimate $\left|\hat{u}_{\omega}(\omega)\right|$. To this end, one assumes that the solution $x_{\omega}(z)$, thought of as function of the complex time $z$, is analytic in the strip $|\operatorname{Im} z|<\tau$, for a suitable $\tau$. Then, by standard arguments (shift of the integration path by $i \tau)$, one gets the estimate

$$
\left|\hat{u}_{\omega}(v)\right| \leqq C^{\prime} e^{-v \tau}, \quad C^{\prime}=\int_{\mathbb{R}}\left|u_{\omega}(t+i \tau)\right| d t,
$$

so that in particular, taking $v=\omega$, one obtains (1.1) with $a=\tau$ and suitable $C$.

This argument is certainly correct, but does not in fact constitute a proof of the exponential dependence of $\Delta E$ on $\omega$. Indeed, it is clear that the quantities $\tau$ and $C$ depend parametrically on $\omega$, and, for example, a dependence of the form $\tau \sim 1 / \omega$ would completely invalidate the exponential dependence of $\Delta E$ on $\omega$. So one still needs a concrete estimate on $\tau$, in order to bound it away from zero; on the other hand, $\tau$ is defined through the analyticity properties of the solution $x_{\omega}(t)$, which is not effectively known.

The approximation usually made (see ref. $[8,9]$ ) is that of replacing the true solution $x_{\omega}(t)$ of (2.2) by a "first order" solution $\xi(t)$, independent of $\omega$, obtained by 
neglecting the interaction, namely, in the present case, the solution of the equation

$$
\ddot{\xi}-e^{-\xi}=0 \text {. }
$$

The general solution of (2.8) can be written explicitly in the form [8]

$$
e^{-\xi(t)}=\frac{v_{0}^{2}}{2\left[\cosh \frac{v_{0}}{2}(t-T)\right]^{2}},
$$

$T$ and $v_{0}$ being free parameters; precisely, $T$ represents the inversion time (defined by $\dot{\xi}(T)=0$ ), while $v_{0}$ is related to the initial data, at some initial time $t_{0}$, by

$$
\frac{1}{2} v_{0}^{2}=\frac{1}{2} \dot{x}_{0}^{2}+e^{-x_{0}}=E_{x}^{0},
$$

$E_{x}^{0}$ being the initial value of $E^{x}$. One easily checks that the right-hand side of (2.9) is analytic in the strip $|\operatorname{Im} z|<\hat{\tau}$, with

$$
\hat{\tau}=\frac{\pi}{v_{0}}
$$

this constant is then taken as an approximation for the coefficient $\tau$ appearing in (2.7). For what concerns the energy exchange, given by (2.6), one thus obtains (1.1) with in general $a=\hat{\tau}$; in particular, for vanishing initial energy of the oscillator, one obtains $a=2 \hat{t}{ }^{1}$ This is the essence of the scheme which we call the Jeans-Landau-Teller method in its heuristic version.

As discussed above, it is clear that such a method requires a careful justification; otherwise, the result given by first order approximation might even be misleading. A justification sometimes adduced to support the above procedure is that the right-hand side of the first of (2.2), being proportional to $y$, is "small" for $\omega$ large; this however is not sufficient because, during the collision, one can only guarantee $\left|y_{\omega}\right| \sim 1 / \omega$, so that the quantity neglected in the approximation turns out to be, so to speak, much larger than the result one wants to prove.

In the present paper we estimate the quantity $\tau$ for the solution of the complete problem (2.2), for the particular case in which the initial energy $E_{0}^{y}$ of the oscillator is negligible. We prove that one has

$$
\tau \geqq \hat{\tau}\left[1-2\left(\frac{v_{0}}{\omega}\right)^{1 / 4}\right],
$$

so that the coefficient $\tau$ turns out to be, for $\omega$ large, as close as one likes to the "first order approximation" $\hat{\tau}$.

\footnotetext{
${ }^{1}$ The same result is also obtained if one takes the average over the phase $\varphi_{\infty}$ appearing in (2.6); this procedure is physically meaningful since (within the approximation used) it corresponds to taking the average over the initial phase of the oscillator, which can be treated, within a statistical approach, as a random variable. So, if one is concerned only with statistical properties, it is sufficient to consider the case of vanishing initial energy. It is not completely clear to us whether the same conclusion holds also independently of the approximation; anyway, this consideration can be a motivation to begin the study of (2.2) in the particular case $E_{0}^{y}=0$
} 


\section{Statement of the Results}

Our main result is contained in the following

Proposition. Consider the system of equations

$$
\ddot{x}=e^{-x}(1+y), \quad \ddot{y}=-\omega^{2} y-e^{-x},
$$

with given initial data $x_{0}, \dot{x}_{0}, y_{0}, \dot{y}_{0}$ at initial time $t_{0}$; denote

$$
E_{0}^{x}=\frac{1}{2} \dot{x}_{0}^{2}+e^{-x_{0}}, \quad E_{0}^{y}=\frac{1}{2}\left(\dot{y}_{0}^{2}+\omega^{2} y_{0}^{2}\right),
$$

and furthermore let $v_{0}, \hat{x}$ and $\hat{\tau}$ be defined by

$$
\frac{1}{2} v_{0}^{2}=E_{0}^{x}, \quad e^{-\hat{x}}=E_{0}^{x}, \quad \hat{\imath}=\frac{\pi}{v_{0}} .
$$

Assume

$$
x_{0} \geqq \hat{x}+2 \hat{\tau} \omega, \quad E_{0}^{y} \leqq v_{0}^{2} e^{-4 \hat{\imath} \omega}, \quad \omega \geqq 2^{8} v_{0} .
$$

Then the solution ${ }^{2} x(z), y(z)$ of system (3.1), as a function of the complex time $z=t+i \sigma$, is analytic in the strip $|\operatorname{Im} z| \leqq \tau$, with

$$
\tau=\hat{\tau}\left[1-2\left(\frac{v_{0}}{\omega}\right)^{1 / 4}\right]
$$

and there satisfies the estimates

$$
\begin{aligned}
\left|e^{-x(t+i \sigma)}\right| & <\frac{1}{8} v_{0}^{2}\left(\frac{v_{0}}{\omega}\right)^{-1 / 2}, \\
|y(t+i \sigma)| & <\frac{1}{2}\left(\frac{v_{0}}{\omega}\right)^{1 / 2}, \\
\left|\int_{t_{0}}^{t}\right| e^{-x(s+i \sigma)}|d s| & <v_{0}\left(\frac{v_{0}}{\omega}\right)^{-1 / 2}, \\
\left|\int_{0}^{ \pm \tau}\right| e^{-x\left(t_{0}+i \sigma\right)}|d \sigma| & <4 v_{0} e^{-2 \tau \omega} ;
\end{aligned}
$$

moreover, one has

$$
\lim _{t \rightarrow \pm \infty}\left|e^{-x(t+i \sigma)}\right|=0, \quad|\sigma| \leqq \tau .
$$

Concerning the assumptions (3.4), let us remark that the third one just establishes a threshold for the "small parameter" $v_{0} / \omega$. In fact, the only really restrictive assumption is the second one, which essentially requires the oscillator to be initially at rest; according to the first assumption, "initially" just refers to any time $t_{0}$ at which the point-mass and the oscillator are sufficiently far apart.

2 The functions $x(t), y(t)$, here introduced, coincide $x_{\omega}(t), y_{\omega}(t)$, appearing in the previous section; the subscript is omitted, from now on, just for notational simplicity 
From this proposition one easily deduces a corollary, which provides a bound for the energy exchange.

Corollary. Within the same notations and assumptions of the above proposition, the energy of the oscillator

$$
E^{y}(t)=\frac{1}{2}\left[\dot{y}^{2}(t)+\omega^{2} y^{2}(t)\right]
$$

satisfies the inequality

$$
\lim _{t \rightarrow \pm \infty}\left|E^{y}(t)-E_{0}^{y}\right|<2 v_{0} \omega e^{-2 \tau \omega} .
$$

As already indicated in Sect. 2, this estimate is obtained by rewriting the second of (3.1) in an integral form, valid for all complex times, which will be used several times in this paper. Introduce the complex time $z=t+i \sigma,|\sigma|<\tau$, and denote

$$
A^{ \pm}(z)=\dot{y}(z) \pm i \omega y(z) ;
$$

then the second of (3.1) is equivalent to

$$
A^{ \pm}(z)=e^{ \pm i \omega\left(z-t_{0}\right)} A\left(t_{0}\right)-\int_{t_{0}}^{z} e^{ \pm i \omega\left(z-z^{\prime}\right)} e^{-x\left(z^{\prime}\right)} d z^{\prime},
$$

with any integration path in the analyticity domain. The obvious relation

$$
y(z)=\frac{1}{2 i \omega}\left(A^{+}(z)-A^{-}(z)\right)
$$

will also be used.

Proof of the Corollary. For real $t$ one has clearly

$$
E^{y}(t)=\frac{1}{2}\left|A^{+}(t)\right|^{2},
$$

so one finds

$$
\left|E^{y}(t)-E_{0}^{y}\right| \leqq \frac{1}{2}\left|\int_{t_{0}}^{t} e^{-i \omega s} e^{-x(s)} d s\right|^{2}+\left|A^{+}\left(t_{0}\right)\right|\left|\int_{t_{0}}^{t} e^{-i \omega s} e^{-x(s)} d s\right| .
$$

Assume, for definiteness, $\omega>0$; shifting the integration path to $\operatorname{Im} s=-\tau$, one can write

$$
\left|\int_{t_{0}}^{t} e^{-i \omega s} e^{-x(s)} d s\right| \leqq \int_{-\tau}^{0}\left|e^{-x\left(t_{0}+i \sigma\right)}\right| d \sigma+e^{-\omega \tau}\left|\int_{t_{0}}^{t}\right| e^{-x(s-i \tau)}|d s|+\int_{-\tau}^{0}\left|e^{-x(t+i \sigma)}\right| d \sigma,
$$

and the result then immediately follows from (3.6c, d), also using (3.7). Q.E.D.

\section{Strategy of Proof, and Two Lemmas}

Throughout the proof we will use the notation

$$
\varepsilon=v_{0} / \omega \text {. }
$$

For definiteness, we shall implicitly refer, in the proof, to the case $\dot{x}_{0}<0$; the case 
$\dot{x}_{0}>0$ (corresponding to a collision occurring before $t_{0}$ ) could be treated in an analogous way.

Concerning the strategy of proof, let us make the preliminary remark that the functions appearing at the right-hand side of (3.1) being entire, the solution $(x(z), y(z))$ is certainly analytic, as a function of the complex time $z$, as long as $e^{-x(z)}$ and $y(z)$ are bounded; thus, it is sufficient to prove the inequalities (3.6) to get, as a byproduct, also the analyticity of the solution.

In turn, the proof of (3.6) is organized as follows: first of all, we prove in a first lemma some preliminary estimates on $e^{-x(t)}$ and $y(t)$, for real $t$; then, in a second lemma, we deduce inequality (3.6d), together with some other useful estimates. Afterwards, we come to the heart of the proof, namely we deduce, at the same time, inequality (3.6b), and two other inequalities, stronger than (3.6a, c), namely:

$$
\begin{gathered}
\left|e^{-x(s+i \sigma)}\right|<\frac{1}{2} v_{0}^{2} \eta(\varepsilon, \sigma), \\
\left|\int_{t_{0}}^{t}\right| e^{-x(s+i \sigma)}|d s|<2 v_{0}(1+4 \varepsilon) \eta(\varepsilon, \sigma),
\end{gathered}
$$

where

$$
\eta(\varepsilon, \sigma)=\frac{1+2 \sqrt{\varepsilon}}{\cos ^{2}\left(\frac{1}{2} \sigma v_{0} \sqrt{1+4 \varepsilon}\right)} .
$$

To see that inequalities (4.2) are indeed stronger than (3.6a, c), it is enough to show that, for $\varepsilon<2^{-8}$ and $\sigma \leqq \tau$, one has

$$
\eta(\varepsilon, \sigma) \leqq \frac{1}{4} \varepsilon^{-1 / 2}
$$

To check (4.4), let us notice that, from $\sigma \leqq \tau=\frac{\pi}{v_{0}}\left(1-2 \varepsilon^{1 / 4}\right)$, one gets

$$
\frac{1}{2} \sigma v_{0} \sqrt{1+4 \varepsilon} \leqq \frac{\pi}{2}-\pi\left(\varepsilon^{1 / 4}-\varepsilon+2 \varepsilon^{5 / 4}\right)
$$

and consequently

$$
\cos \left(\frac{1}{2} \sigma v_{0} \sqrt{1+4 \varepsilon}\right) \geqq \sin \pi\left(\varepsilon^{1 / 4}-\varepsilon+2 \varepsilon^{5 / 4}\right) .
$$

It then easily follows that, for $\varepsilon$ satisfying the above assumption, the right-hand side is larger than $2 \varepsilon^{1 / 4} \sqrt{1+2 \sqrt{\varepsilon}}$; inequality (4.4) is then immediately obtained.

In the course of the proof we shall use in several occasions a technique, sometimes called the "bootstrap method," which is similar in a sense to the one based on the induction principle in the discrete case. It goes as follows: let $f$ be a continuous function of the real variable $s$, and suppose one wants to prove an inequality of the form $f(s)<K$, in a closed interval $I$ (possibly coinciding with the whole real axis). To this end it is sufficient to prove that: (i) there exists a point $s_{0} \in I$, such that $f\left(s_{0}\right)<K$; (ii) in any closed interval $\left[s_{0}, s_{1}\right] \subset I$ (or $\left[s_{1}, s_{0}\right]$ if 
$\left.s_{1}<s_{0}\right)$, the weak inequality $f(s) \leqq K$ implies the strict inequality $f(s)<K$. The proof is totally elementary: assume, in contradiction, that the strict inequality is not satisfied, for example, for some $s>s_{0}$; let $s_{1}>s_{0}$ be the first value of $s$, such that $f\left(s_{1}\right)=K$. Since in the interval $\left[s_{0}, s_{1}\right]$ one has $f(s) \leqq K$, one deduces from (ii) $f\left(s_{1}\right)<K$, which is a contradiction. The method clearly applies also in the case of more than one simultaneous inequalities.

In the remaining part of this section we state and prove the two lemmas mentioned above.

Lemma 1. Consider the system of equations (3.1), and assume

$$
E \leqq v_{0}^{2}, \quad \varepsilon \leqq \frac{1}{16},
$$

where $E$ denotes the total energy, $E=E^{x}+E^{y}+y e^{-x}$, and $\varepsilon$ is defined by (4.1). Then for any $t \in \mathbb{R}$ one has

$$
\begin{aligned}
|y(t)| & <\sqrt{2} \varepsilon, \\
\left|E^{x}(t)-\frac{1}{2} v_{0}^{2}\right| & <2 v_{0}^{2} \varepsilon
\end{aligned}
$$

and moreover

$$
\int_{-\infty}^{\infty} e^{-x(t)} d t<2 v_{0}(1+4 \varepsilon)
$$

Proof. Both inequalities (4.6) are easily proven by the bootstrap method. Concerning the former one, one immediately deduces, from (4.5),

$$
\left|y\left(t_{0}\right)\right|<\varepsilon\left(\sqrt{1+\frac{1}{4} \varepsilon^{2}}+\frac{1}{2} \varepsilon\right)<\sqrt{2} \varepsilon .
$$

Take now any $t_{1}$, for example $t_{1}>t_{0}$, and assume that in the interval $\left[t_{0}, t_{1}\right]$ the inequality $|y(t)| \leqq \sqrt{2} \varepsilon$ holds. Then one has $(1+y) e^{-x}>0$, and the strict inequality (4.6a), in the same interval, immediately follows from energy conservation. Then (4.6a) holds for any $t \in \mathbb{R}$.

As a byproduct of inequality (4.6a), one deduces that there is just one inversion time, i.e. a time $\hat{t}$ such that $\dot{x}(\hat{t})=0$; indeed, this immediately follows from

$$
\ddot{x}=e^{-x}(1+y)>0 \text {. }
$$

Let us now come to (4.6b), which is obviously satisfied for $t=t_{0}$. Assume then $\left|E^{x}(t)-\frac{1}{2} v_{0}^{2}\right| \leqq 2 v_{0}^{2} \varepsilon$, up to some $t_{1}$; one immediately gets

$$
e^{-x(t)} \leqq E^{x}(t) \leqq \frac{1}{2} v_{0}^{2}(1+4 \varepsilon)<\frac{v_{0}^{2}}{\sqrt{2}}
$$

(for the last inequality, the assumption (4.5) on $\varepsilon$ is fairly sufficient). On the other hand, from the energy theorem applied to the equation of motion for $x$, one can write

$$
E^{x}(t)-E^{x}\left(t_{0}\right)=\int_{t_{0}}^{t} y(s) e^{-x(s)} \dot{x}(s) d s
$$


considering, for definiteness, the case $t_{0}<\hat{t}<t$ (which turns out to be the worst one), one then gets

$$
\begin{aligned}
\left|E^{x}(t)-E^{x}\left(t_{0}\right)\right| & \leqq \sup _{s \in \mathbb{R}}|y(s)|\left[\int_{t_{0}}^{\hat{t}} e^{-x(s)}(-\dot{x}(s)) d s+\int_{\hat{t}}^{t} e^{-x(s)} \dot{x}(s) d s\right] \\
& \leqq \sup _{s \in \mathbb{R}}|y(s)|\left(2 e^{-x(\hat{t})}-e^{-x\left(t_{0}\right)}-e^{-x(t)}\right) .
\end{aligned}
$$

Using (4.8) and (4.6a) one has thus $\left|E^{x}(t)-E^{x}\left(t_{0}\right)\right|<2 v_{0}^{2} \varepsilon$, and inequality (4.6b) follows.

Finally, concerning estimate (4.7), one first writes the obvious inequality

$$
\left|\dot{x}\left(t^{\prime}\right)-\dot{x}\left(t^{\prime \prime}\right)\right|=\left|\int_{t^{\prime}}^{t^{\prime \prime}} e^{-x(t)}(1+y(t)) d t\right| \geqq\left(1-\sup _{t \in \mathbb{R}}|y(t)|\right) \int_{t^{\prime}}^{t^{\prime \prime}} e^{-x(t)} d t,
$$

for any pair $t^{\prime}, t^{\prime \prime}$, with $t^{\prime}<t^{\prime \prime}$; on the other hand, one has

$$
\begin{aligned}
\left|\dot{x}\left(t^{\prime}\right)-\dot{x}\left(t^{\prime \prime}\right)\right| & \leqq 2 \sup _{t \in \mathbb{R}}|\dot{x}(t)| \leqq 2 \sup _{t \in \mathbb{R}} \sqrt{2 E^{x}(t)} \\
& <2 v_{0} \sqrt{1+4 \varepsilon}
\end{aligned}
$$

and finally

$$
\int_{t^{\prime}}^{t^{\prime \prime}} e^{-x(t)} d t \leqq \frac{\left|\dot{x}\left(t^{\prime}\right)-\dot{x}\left(t^{\prime \prime}\right)\right|}{1-\sup _{t \in \mathbb{R}}|y(t)|}<2 v_{0} \frac{\sqrt{1+4 \varepsilon}}{1-\sqrt{2} \varepsilon}
$$

using assumption (4.5) on $\varepsilon$, inequality (4.7) follows immediately. This concludes the proof of the lemma.

Q.E.D.

Lemma 2. Under the assumptions (3.4) of the Proposition, one has

$$
\begin{aligned}
& \left|e^{-x\left(t_{0}+i \sigma\right)}\right|<v_{0}^{2} e^{-2 \hat{\tau} \omega}, \\
& \left|y\left(t_{0}+i \sigma\right)\right|<\varepsilon
\end{aligned}
$$

for any $\sigma$ with $|\sigma| \leqq \hat{\tau}=\pi / v_{0}$, and moreover

$$
\left|\int_{0}^{ \pm \hat{\tau}}\right| e^{-x\left(t_{0}+i \sigma\right)}|d \sigma|<4 v_{0} e^{-2 \hat{\tau} \omega} .
$$

Proof. The pair of inequalities (4.9) is proven by the bootstrap method, with respect to the real variable $\sigma$ in the interval $[-\hat{\tau}, \hat{\tau}]$, using the fact that they are true for $\sigma=0$. Take any $\sigma_{1}$ with $\left|\sigma_{1}\right| \leqq \hat{\tau}$, say $\sigma_{1}>0$, and assume both inequalities (4.9) are satisfied for $0 \leqq \sigma \leqq \sigma_{1}$, with " $\leqq$ " in place of " $<$."

Consider first inequality (4.9b). From the expression (3.12) for $y$, we can write

$$
\begin{aligned}
\left|y\left(t_{0}+i \sigma\right)\right| \leqq & \frac{1}{2 \omega}\left[\left|A^{+}\left(t_{0}\right)\right| e^{-\omega \sigma}+\int_{0}^{\sigma} e^{-\left(\sigma-\sigma^{\prime}\right) \omega}\left|e^{-x\left(t_{0}+i \sigma^{\prime}\right)}\right| d \sigma^{\prime}\right. \\
& \left.+\left|A^{-}\left(t_{0}\right)\right| e^{+\omega \sigma}+\int_{0}^{\sigma} e^{+\left(\sigma-\sigma^{\prime}\right) \omega}\left|e^{-x\left(t_{0}+i \sigma^{\prime}\right)}\right| d \sigma^{\prime}\right] .
\end{aligned}
$$


Recalling that $\left|A^{ \pm}\left(t_{0}\right)\right| \leqq \sqrt{2} v_{0} e^{-2 \hat{\tau} \omega}$ by hypothesis, and that (4.9a) is assumed to hold with " $\leqq$," then one has $\left|y\left(t_{0}+i \sigma\right)\right| \leqq 2 \varepsilon e^{-2 \tau \omega} e^{-(\tau-\sigma) \omega}<\varepsilon$, so that the (4.9b) is achieved.

In order to prove (4.9a), one has to estimate $\operatorname{Re} x\left(t_{0}+i \sigma\right)$. By twice integrating the first of (3.1), using also $\operatorname{Im} \dot{x}\left(t_{0}\right)=0$, one finds

$$
\operatorname{Re} x\left(t_{0}+i \sigma\right)=x\left(t_{0}\right)-\int_{0}^{\sigma} d \sigma^{\prime} \int_{0}^{\sigma^{\prime}} \operatorname{Re}\left(e^{-x\left(t_{0}+i \sigma^{\prime \prime}\right)}\left[1+y\left(t_{0}+i \sigma^{\prime \prime}\right)\right]\right) d \sigma^{\prime \prime} .
$$

Then one gets

$$
\left|\operatorname{Re} x\left(t_{0}+i \sigma\right)\right|>x\left(t_{0}\right)-2 \pi^{2} e^{-2 \hat{\tau} \omega}>x\left(t_{0}\right)-\ln 2,
$$

which gives immediately the desired inequality.

Finally, inequality (4.10) is proven by a direct computation, namely

$$
\int_{0}^{\hat{\tau}}\left|e^{-x\left(t_{0}+i \sigma\right)}\right| d \sigma \leqq \hat{\tau} \sup _{0<\sigma \leqq \hat{\tau}}\left|e^{-x\left(t_{0}+i \sigma\right)}\right| \leqq \pi v_{0} e^{-2 \hat{\tau} \omega} .
$$

\section{Proof of the Proposition}

As remarked above, in order to prove the proposition we have to prove, for $|\sigma| \leqq \tau$, inequalities (4.2), (3.6b, d) and (3.8). In fact, (3.6d) turns out to be weaker than the already established inequality (4.10), and so is already proven.

Now we concentrate on (4.2) and (3.6b), using the bootstrap method; by Lemma 2 , they are clearly satisfied for $t=t_{0}$. So, let us assume they are satisfied with " $\leqq$ " in place of " $<$," in some interval $\left[t_{0}, t_{1}\right]$, and prove that in the same interval the strict inequalities hold. For the case of (3.6b), the proof is a consequence of the following

Lemma 3. Under the assumptions (3.4) of the proposition, if inequalities (4.2) and (3.6b) hold with " $\leqq$ " in place of " $<$ " in a closed interval $\left[t_{0}, t_{1}\right]$, then the strict inequality (3.6b) holds there, and for any $t$ in that interval one has

$$
\int_{0}^{\sigma}\left|y\left(t+i \sigma^{\prime}\right) e^{-x\left(t+i \sigma^{\prime}\right)}\right| d \sigma^{\prime}<\frac{3}{32} v_{0} \varepsilon
$$

Proof. By (3.11) and (3.12) we can write

$$
y\left(t_{0}+i \sigma\right)=\frac{1}{2 i \omega}\left[A^{+}\left(t_{0}\right) e^{i \omega\left(z-t_{0}\right)}-I_{1}(z)-A^{-}\left(t_{0}\right) e^{-i \omega\left(z-t_{0}\right)}+I_{2}(z)\right],
$$

where $z=t+i \sigma$,

$$
\begin{aligned}
& I_{1}(z)=\int_{t_{0}}^{z} e^{-i \omega\left(z-z^{\prime}\right)} e^{-x\left(z^{\prime}\right)} d z^{\prime} \\
& I_{2}(z)=\int_{t_{0}}^{z} e^{+i \omega\left(z-z^{\prime}\right)} e^{-x\left(z^{\prime}\right)} d z^{\prime},
\end{aligned}
$$

and the integration paths are inside the rectangle $|\sigma| \leqq \tau, t \in\left[t_{0}, t_{1}\right]$. 
For definiteness let us consider the case $\sigma>0$. In the first integral $I_{1}$ we choose the integration path $\gamma$ as the union of the real interval $\gamma^{\prime}=\left[t_{0}, t\right]$ and the "vertical" interval $\gamma^{\prime \prime}=[t, t+i \sigma]$. Correspondingly we have $I_{1}=I_{1}^{\prime}+I_{1}^{\prime \prime}$, and we find the estimates

$$
\begin{aligned}
& \left|I_{1}^{\prime}(z)\right| \leqq e^{-\omega \sigma} \int_{t_{0}}^{t} e^{-x(s)} d s<2 v_{0}(1+4 \varepsilon) e^{-\sigma \omega} \\
& \left|I_{1}^{\prime \prime}(z)\right| \leqq \int_{0}^{\sigma} e^{-\omega\left(\sigma-\sigma^{\prime}\right)}\left|e^{-x\left(t+i \sigma^{\prime}\right)}\right| d \sigma^{\prime}<\frac{1}{2} v_{0} \varepsilon \eta(\varepsilon, \sigma)
\end{aligned}
$$

which are established using Estimate (4.7) of Lemma 1 for the first one, and (4.2a) for the second one.

In the second integral $I_{2}$ we choose instead the integration path $\gamma$ as the union of the "vertical ascending" interval $\gamma^{\prime}=\left[t_{0}, t_{0}+i \tau\right]$, the "horizontal" interval $\gamma^{\prime \prime}=\left[t_{0}+i \tau, t+i \tau\right]$, and the "vertical descending" interval $\gamma_{3}=[t+i \tau, t+i \sigma]$. Correspondingly we have $I_{2}=I_{2}^{\prime}+I_{2}^{\prime \prime}+I_{2}^{\prime \prime \prime}$, and we find the estimates

$$
\begin{aligned}
\left|I_{2}^{\prime}(z)\right| & \leqq \int_{0}^{\tau} e^{\omega\left(\sigma-\sigma^{\prime}\right)}\left|e^{-x\left(t_{0}+i \sigma^{\prime}\right)}\right| d \sigma^{\prime}<v_{0}^{2} e^{-\tau \omega}, \\
\left|I_{2}^{\prime \prime}(z)\right| & \leqq \int_{t_{0}}^{t} e^{-\omega(\tau-\sigma)}\left|e^{-x(s+i \tau)}\right| d s<2 v_{0}(1+4 \varepsilon) e^{-\omega(\tau-\sigma)} \eta(\varepsilon, \tau), \\
\left|I_{2}^{\prime \prime \prime}(z)\right| & \leqq \int_{\sigma}^{\tau} e^{\omega\left(\sigma-\sigma^{\prime}\right)}\left|e^{-x\left(t+i \sigma^{\prime}\right)}\right| d \sigma^{\prime}<\frac{1}{2} v_{0} \varepsilon \eta(\varepsilon, \tau),
\end{aligned}
$$

which are established using Estimate (4.9a) of Lemma 2 for the first one, (4.2b) for the second one, and (4.2a) for the third one.

Moreover, from Assumptions (3.4) we have

$$
\left|A^{+}\left(t_{0}\right) e^{i \omega z}+A^{-}\left(t_{0}\right) e^{-i \omega z}\right|<2 v_{0} e^{-\tau \omega},
$$

so that, collecting terms, one gets

$$
\begin{aligned}
|y(t+i \sigma)| \leqq & \frac{1}{2} \varepsilon\left[2(1+4 \varepsilon) e^{-\sigma \omega}+\varepsilon \eta(\varepsilon, \tau)+(2+\varepsilon) e^{-\tau \omega}\right. \\
& \left.+2(1+4 \varepsilon) \eta(\varepsilon, \tau) e^{-\omega(\tau-\sigma)}\right] .
\end{aligned}
$$

This inequality implies immediately inequality (3.6b), as claimed.

Coming to inequality (5.1), one first writes

$$
\int_{0}^{\sigma}\left|y\left(t+i \sigma^{\prime}\right) e^{-x\left(t+i \sigma^{\prime}\right)}\right| d \sigma^{\prime} \leqq \frac{1}{2} v_{0}^{2} \eta(\varepsilon, \sigma) \int_{0}^{\sigma}\left|y\left(t+i \sigma^{\prime}\right)\right| d \sigma^{\prime} .
$$

One then makes use of (5.4), as well as the bootstrap assumption for $\eta$; noticing that the dominant term in right-hand side of (5.4) is the last one, and that the integration over $\sigma$ gives an extra factor $\omega^{-1}$, the result is easily found.

Q.E.D.

Coming back to the proof of the proposition, we have to show that (4.2) are also satisfied as strict inequalities in the interval $\left[t_{0}, t_{1}\right]$. The strategy of proof is the 
following: for any $t \in\left[t_{0}, t_{1}\right]$, we introduce a convenient approximation $\xi_{t}(z)$ (described below) to the solution $x(z)$, and prove that: i) $\xi_{t}$ satisfies the inequalities

$$
\begin{aligned}
\left|e^{-\xi_{t}(t+i \sigma)}\right| & \leqq \frac{1}{2} v_{0}^{2} \frac{\eta(\varepsilon, \sigma)}{1+\sqrt{\varepsilon}} \\
\int_{t_{0}}^{t}\left|e^{-\xi_{t}(s+i \sigma)}\right| d s & \leqq 2 v_{0}(1+4 \varepsilon) \frac{\eta(\varepsilon, \sigma)}{1+\sqrt{\varepsilon}} ;
\end{aligned}
$$

ii) one has

$$
\left|e^{-x(t+i \sigma)}\right|<(1+\sqrt{\varepsilon})\left|e^{-\xi_{t}(t+i \sigma)}\right| .
$$

This is obviously enough for the proof of (4.2).

As to the approximating function $\xi_{t}(z)$, for any $t \in\left[t_{0}, t_{1}\right]$ this is defined as the solution of the approximated equation (2.8), with initial data at time $z=t$, given by $\xi_{t}(t)=x(t), \dot{\xi}_{t}(t)=\dot{x}(t)$. Loosely speaking, this corresponds to following the true equations (3.1) from $t_{0}$ to $t$, and then switching to the approximate equation (2.8) from $t$ to $z$; in fact the approximation $\xi_{t}(z)$, although defined for any $z$, will be used only for $z=t+i \sigma$. In analogy with (2.9), the explicit expression of $\xi_{t}(z)$ is given by

$$
e^{-\xi_{t}(z)}=\frac{v_{t}^{2}}{2 \cosh ^{2} \frac{v_{t}}{2}\left(z-T_{t}\right)},
$$

with $v_{t}=\sqrt{2 E^{x}(t)}$ and a suitable $T_{t}$.

From the expression (5.7), inequalities (5.5) follow rather easily. Indeed one has

$$
\begin{aligned}
\left|e^{-\xi_{t}(t+i \sigma)}\right| & =\frac{v_{t}^{2}}{2 \cosh ^{2} \frac{v_{t}}{2}\left(t-T_{t}\right)-2 \sin ^{2} \frac{v_{t}}{2} \sigma} \\
& \leqq \frac{e^{-\xi_{t}(t)}}{\cos ^{2} \frac{v_{t}}{2} \sigma}=\frac{e^{-x(t)}}{\cos ^{2} \frac{v_{t}}{2} \sigma} ;
\end{aligned}
$$

from this, using $e^{-x(t)} \leqq E^{x}(t)$ and the estimate $E^{x}(t) \leqq \frac{1}{2} v_{0}^{2}(1+4 \varepsilon)$, which follows from (4.6b) of Lemma 1, one gets the former of (5.5); the latter too easily follows from (5.8), using (4.7) of Lemma 1.

Inequality (5.6) requires instead some more work. Let us denote

$$
\delta_{t}(z)=x(z)-\xi_{t}(z)
$$

then one has $\ddot{\delta}_{t}=e^{-\xi_{t}}\left(e^{-\delta_{t}}-1\right)+y e^{-x}$, or also, emphasizing the linear part,

$$
\ddot{\delta}_{t}=-e^{-\xi_{t}} \delta_{t}+e^{-\xi_{t}}\left(e^{-\delta_{t}}-1+\delta_{t}\right)+y e^{-x} .
$$

This is a non-autonomous differential equation for $\delta_{t}$, with initial data (at time $z=t) \delta_{t}(t)=\dot{\delta}_{t}(t)=0$, as follows from the very definition of $\xi_{t}$. With these initial data, Eq. (5.10) can be rewritten in the integral form

$$
\delta_{t}(z)=\int_{t}^{z} G(z, w) F(w) d w,
$$


where

$$
F(w)=e^{-\xi_{t}(w)}\left(e^{-\delta_{t}(w)}-1+\delta_{t}(w)\right)+y(w) e^{-x(w)},
$$

while $G(z, w)$ is a family of solutions of the linearized equation

$$
\ddot{\delta_{t}}=-e^{-\xi_{t}} \delta_{t}
$$

depending on the parameter $w$, such that $G(w, w)=0, \dot{G}(w, w)=1$. It is not difficult to check that $G$ is given by

$$
\begin{aligned}
G(z, w) & =\frac{4}{v_{t}^{2}}[(z-w) g(z) g(w)+g(z)-g(w)], \\
g(z) & =\frac{v_{t}}{2} \tanh \frac{v_{t}}{2}\left(z-T_{t}\right)
\end{aligned}
$$

for $z=t+i \sigma, w=t+i \mu$; after some work one finds

$$
\left|\delta_{t}\right|<2\left|\delta_{t}\right|^{2} \varepsilon^{-1 / 2}+\frac{9}{32} \varepsilon^{3 / 4} .
$$

The details of this computation are not fully reported here; the essential steps are the following:

i) After writing (say for positive $\sigma$ )

$$
\begin{aligned}
\left|\delta_{t}(t+i \sigma)\right| \leqq & \int_{0}^{\sigma}\left|G(t+i \sigma, t+i \mu) e^{-\xi_{t}(t+i \mu)}\right|\left|e^{-\delta_{t}(t+i \mu)}-1+\delta_{t}(t+i \mu)\right| d \mu \\
& +\int_{0}^{\sigma}|G(t+i \sigma, t+i \mu)|\left|y(t+i \mu) e^{-x(t+i \mu)}\right| d \mu
\end{aligned}
$$

one makes for $\delta_{t}$ the bootstrap assumption $\left|\delta_{t}(t+i \mu)\right| \leqq \log 2$, which leads to the estimate $\left|e^{-\delta_{t}(t+i \mu)}-1+\delta_{t}(t+i \mu)\right|<\left|\delta_{t}^{2}\right|$.

ii) By direct computation one gets the estimate

$$
\int_{0}^{\sigma}\left|G(t+i \sigma, t+i \mu) e^{-\xi_{t}(t+i \mu)}\right| d \mu \leqq 8 \tan ^{2} \frac{v_{t}}{2} \sigma ;
$$

the first term in (5.15) is then properly estimated by the first term in (5.14).

iii) By direct computation one gets the estimate

$$
\sup _{0 \leqq \mu \leqq \sigma}|G(t+i \sigma, t+i \mu)| \leqq \frac{6}{v_{t}} \tan \frac{v_{t}}{2} \sigma ;
$$

by means of (5.1) of Lemma 3 one then concludes that the second term in (5.15) is also properly estimated by the second term of (5.14).

From (5.14), taking into account assumption (3.4), one immediately gets

$$
\left|\delta_{t}\right| \leqq \frac{1}{4} \varepsilon^{1 / 2}
$$

which on the one hand provides the consistency of the bootstrap procedure, and on the other hand leads immediately to (5.6). As already remarked, this is enough to conclude the proof of inequalities (3.6). 
To complete the proof of the proposition, we only need to prove (3.7). It would be rather easy to generalize Lemma 2 , and show that $\left|e^{-x(t+i \sigma)}\right|$ decays to zero exponentially, for $t \rightarrow \pm \infty$; in fact, the simple property that the limit vanishes is an easy consequence of (3.6c), according to which the function $e^{-x(t+i \sigma)}$ is summable (one must also take into account that, in the analyticity strip, the derivative of $e^{-x(z)}$ is bounded).

Q.E.D.

\section{Conclusions}

We have shown that, for a particular model of interest in the domain of atomic collisions, the Jeans-Landau-Teller method can be made rigorous, so that the exponential law (3.9) for the energy exchanges is justified; moreover, the explicit estimate (3.5) found for the coefficient for the width of the analyticity strip $\tau$ shows that this quantity approaches, for $\omega$ large, the value $\hat{\tau}$ which is obtained in the heuristic version of the method. Numerical computations, to be reported soon [6], show that this result is, in a sense, optimal: namely, it appears that the distance of the closest singularity of $e^{-x(z)}$ to the real axis tends to $\hat{\tau}$ (from below) for increasing $\omega$, although faster than estimate (3.5).

On the other hand, one should also stress that we were able to prove such properties only under a very restrictive hypothesis (usually assumed also in the heuristic treatments), namely that before collision the oscillator has negligible energy. This assumption is essential in our approach, because without it the amplitudes $A^{ \pm}(z)$ of the oscillations cannot be kept controlled outside the real axis; indeed, as could be seen within perturbation theory, $A^{ \pm}(z)$ does actually increase exponentially with $\operatorname{Im} z{ }^{3}$ However, this fact does not imply that, for a nonvanishing initial amplitude, the Jeans-Landau-Teller method becomes useless: rather, it indicates that some essential changes in the strategy of the proof are needed; work is in progress in this direction. In fact, numerical computations [6] seem to indicate that the exponential law (1.1) persists, with apparently no essential changes, even for nonvanishing initial amplitudes.

Other problems remain open. Let us just quote the extension of the present treatment to the case of a general class of potentials, and moreover to systems of several oscillators. Finally, as anticipated in the Introduction, one has the problem of establishing a connection between the present method and the general methods of perturbation theory. We hope to be able to give some results about these problems in the future.

\section{Appendix}

In this appendix we outline an elementary physical interpretation of the model studied in this paper; we follow almost literally the original treatment of Rapp [8];

\footnotetext{
3 The deep reason is that the canonical transformations entering perturbation theory contain Fourier terms of the form $e^{i k \vartheta}$; during the motion one has $\vartheta \simeq \vartheta_{0}+\omega z$, so that these terms increase exponentially with $\operatorname{Im} z$
} 
only, we'choose to adapt the language to the case of the collision of a diatomic molecule with a fixed wall, while Rapp considers the collision of a point mass with the molecule (for an illustration, see the Fig. 1).

Denote by $M=m_{1}+m_{2}$, and $\mu=\frac{m_{1} m_{2}}{m_{1}+m_{2}}$, the total mass of the molecule and the reduced mass respectively, by $X$ the coordinate of the center of mass, and by $Y$ the relative coordinate $x_{2}-x_{1}$. The Hamiltonian has the form

$$
H=\frac{P_{X}^{2}}{2 M}+\frac{P_{Y}^{2}}{2 \mu}+\frac{\mu \Omega^{2}}{2}\left(Y-Y_{0}\right)^{2}+V(X, Y),
$$

where $\Omega$ represents the proper frequency of the molecule, $Y_{0}$ is its rest length, and finally, $V(X, Y)$ is a suitable potential, which models the interaction of the molecule with the wall.

Following Rapp, one first assumes that $V$ depends on the distance $r=X-\frac{m_{2}}{M} Y$ between the wall and the closer atom, and follows the very simple law $V=V_{0} e^{-r / r_{0}}$, with suitable constants $V_{0}$ and $r_{0}$; one has then

$$
\begin{aligned}
V & =V_{0}^{\prime} e^{-\left[X-\frac{m_{2}}{M}\left(Y-Y_{0}\right)\right] / r_{0}} \\
& =V_{0}^{\prime} e^{-X / r_{0}}\left(1+\frac{m_{0}}{M r_{0}}\left(Y-Y_{0}\right)+\ldots\right),
\end{aligned}
$$

with $V_{0}^{\prime}=V_{0} e^{m_{2} Y_{0} / M r_{0}}$. Making a crude physical approximation, one then neglects the higher order terms in the expansion of the exponential, and writes the (second order) equations of motion

$$
\begin{aligned}
& \ddot{X}=\frac{V_{0}^{\prime}}{M r_{0}} e^{-X / r_{0}}\left[1+\frac{m_{2}}{M r_{0}}\left(Y-Y_{0}\right)\right], \\
& \ddot{Y}=-\Omega^{2}\left(Y-Y_{0}\right)+\frac{V_{0}^{\prime}}{m_{1} r_{0}} e^{-X / r_{0}} .
\end{aligned}
$$

Finally, one eliminates some dimensional constants by suitably rescaling $X, Y$ and the time $t$, namely posing $X=r_{0} x, Y=Y_{0}+\frac{M r_{0}}{m_{2}} y$ and $t^{\prime}=\sqrt{\frac{M r_{0}^{2}}{V_{0}^{\prime}}} t$ (notice that all

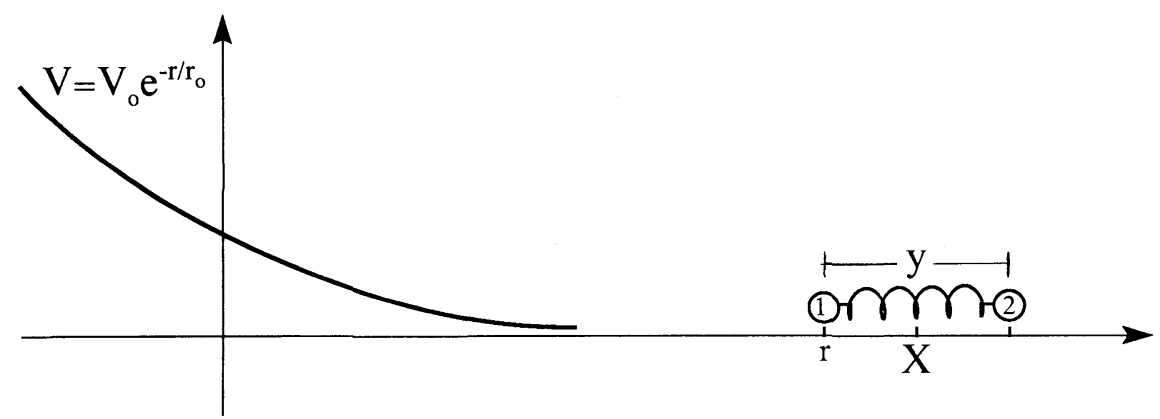

Fig. 1. Illustrating the model 
variables are now dimensionless). For the particular case $m_{1}=m_{2}$, one gets for $x, y$ precisely the system of equations (2.2), with $\omega=\sqrt{\frac{M r_{0}^{2}}{V_{0}^{\prime}}} \Omega$; these are canonical equations, with Hamiltonian (2.1).

Acknowledgements. We are grateful to P. Tosi (Trento), for having drawn our attention to the paper by Rapp, and for some useful discussions. We are also indebted to G. Gallavotti (Roma) and A. Giorgilli (Milano) for very helpful comments.

\section{References}

1. Neishtadt, A.I.: Prikl. Matem. Mekan. 45, 80 (1981) [PMM U.S.S.R. 45, 58 (1982)]

2. Benettin, G., Galgani, L., Giorgilli, A.: Commun. Math. Phys. 113, 87 (1987)

3. Benettin, G., Galgani, L., Giorgilli, A.: Commun. Math. Phys. 121, 557 (1989)

4. Nekhoroshev, N.N.: Usp. Mat. Nauk 32 (1977) [Russ. Math. Surv. 32, 1 (1977)]

5. Nekhoroshev, N.N.: Trudy Sem. Petrows. No. 5,5 (1979) [Topics in Modern Mathematics, Petrovskii Sem. no. 5, Oleinik, O.A. (ed.) New York: Consultant Bureau 1985]

6. Benettin, G., Carati, A., Sempio, P.: paper in preparation

7. Rapp, D., Kassal, T.: Chem. Rev. 65, 61 (1969)

8. Rapp, D.: J. Chem. Phys. 32, 735 (1960)

9. O’Neil, T.M., Hjorth, P.G.: Phys. Fluids 28, 3241 (1985); O'Neil, T.M., Hjorth, P.G., Beck, B., Fajans, J., Malmberg, J.H.: Collisional Relaxation of Strongly Magnetized Pure Electron Plasma (Theory and Experiment) In: Strongly Coupled Plasma Physics. Proceedings of the Yamada Conference No. 24, Japan, p. 313, North-Holland: Amsterdam 1990

10. Jeans, J.H.: Phil. Mag. 6, 279 (1903)

11. Jeans, J.H.: Phil. Mag. 10, 91 (1905)

12. Landau, L., Teller, E.: Physik. Z. Sowjetunion 10, 34 (1936), In: Ter Haar D. (ed.) Collected Papers of L.D. Landau, p. 147, Oxford: Pergamon Press 1965

Communicated by J.-P. Eckmann 